\title{
Preterm human milk composition: a systematic literature review
}

\author{
Catherine Boyce ${ }^{1}$, Mistral Watson ${ }^{1}$, Grace Lazidis ${ }^{1}$, Sarah Reeve ${ }^{1}$, Kenneth Dods ${ }^{2}$, Karen Simmer ${ }^{3}$ \\ and Gemma McLeod ${ }^{3 *}$ \\ ${ }^{1}$ Faculty of Medicine, Dentistry and Health Sciences, The University of Western Australia, 35 Stirling Highway, Crawley, \\ WA 6009, Australia \\ ${ }^{2}$ ChemCentre, Food and Compositional Chemistry, Building 500 Manning Road, Bentley, WA 6101, Australia \\ ${ }^{3}$ School of Paediatrics and Child Health, The University of Western Australia, and Women and Newborn Health Service, \\ 374 Bagot Road, Subiaco, WA 6008, Australia
}

(Submitted 17 November 2015 - Final revision received 9 July 2016 - Accepted 14 July 2016 - First published online 15 August 2016)

\section{Abstract}

There are wide variations in the macronutrient values adopted by neonatal intensive care units and industry to fortify milk in efforts to achieve recommended intakes for preterm infants. Contributing to this is the variation in macronutrient composition of preterm milk between and within mothers and the variable quality of milk analyses used to determine the macronutrient content of milk. We conducted a systematic review of the literature using articles published in English between 1959 and 2013 that reported the concentrations of one or more macronutrients or energy content in human preterm milk, sampled over a representative 24-h period. Searched medical databases included Ovid Medline, Scopus, CINAHL and the Cochrane Library. Results are presented as mean values and ranges for each macronutrient during weeks 1-8 of lactation, and preferred mean values $(\mathrm{g} / 100 \mathrm{ml})$ for colostrum (week 1 ) and mature milk (weeks 2-8; protein: $1 \cdot 27$, fat: 3.46, lactose: 6.15 and carbohydrate: 7·34), using data from studies employing the highest-quality analyses. Industry-directed fortification practices using these mean values fail to meet protein targets for infants weighing $<1000 \mathrm{~g}$ when the fortified milk is fed $<170-190 \mathrm{ml} / \mathrm{kg}$ per $\mathrm{d}$, and the protein:energy ratio of the fortified milk is inadequate. This study aimed to provide additional information to industry in order to guide their future formulation of breast milk fortifiers. Quality macronutrient analyses of adequately sampled preterm breast milk would improve our understanding of the level of fortification needed to meet recommended protein and energy intakes and growth targets, as well as support standardised reporting of nutritional outcomes.

\section{Key words: Preterm infants: Breast milk: Composition: Macronutrients: Fortification}

Compared with healthy term infants, preterm infants are born physiologically immature, nutritionally compromised, growth restricted and at risk of long-term complications ${ }^{(1-3)}$. The optimal nutrition for preterm infants is to be fed human breast milk $^{(4,5)}$, and to mirror the growth and development of the age-matched healthy fetus. However, the nutrient and energy contents of native breast milk are insufficient to meet the needs of the preterm infant ${ }^{(5-7)}$, and fortification processes are thus used to assist in achieving the latest European recommended intakes $^{(8)}$. These recommendations target $460-565 \mathrm{~kJ} / \mathrm{kg}$ per $\mathrm{d}$ (110-135 kcal $/ \mathrm{kg}$ per d), with $4.0-4.5 \mathrm{~g}$ protein $/ \mathrm{kg}$ per $\mathrm{d}$ (3.6- $4.1 \mathrm{~g}$ protein $/ 418.4 \mathrm{~kJ}(100 \mathrm{kcal}))$ for preterm infants weighing $<1000 \mathrm{~g}$ and $3.5-4.0 \mathrm{~g}$ protein $/ \mathrm{kg}$ per d $(3.2-3.6 \mathrm{~g}$ protein $/ 418.4 \mathrm{~kJ}$ (100 kcal)) for infants weighing between 1000 and $1800 \mathrm{~g}^{(8)}$.

Common practice is to fortify on an assumed average preterm breast milk composition ${ }^{(6,7,9)}$; however, significant variation in the macronutrient content of breast milk exists between and within mothers ${ }^{(6,10-22)}$, reflected by wide variations in macronutrient values derived by studies and adopted by neonatal intensive care units and industry to fortify milk, raising concerns that this practice may lead to undernourishment of some infants and over-nourishment of others ${ }^{(7)}$. The lack of a globally accepted reference 'assumed' preterm breast milk composition inhibits standardised reporting of nutritional intakes, making it difficult for clinicians and researchers to assess the adequacy of nutritional intakes and the role of nutrition in growth and developmental preterm outcomes.

Macronutrient composition, particularly lipid concentration, is dependent on the method of sampling, stage of lactation, gestational age, maternal diet, presence of maternal infection and parity ${ }^{(13,23)}$. In addition, there is significant diurnal and inter-feed variation; as such, study designs that incorporate milk expressions collected at each feed over a 24 -h period are preferred to ensure that analysis is being undertaken on a representative milk sample ${ }^{(24)}$. Accurately measuring the composition of individual breast milk feeds is difficult, costly and time consuming, and studies have collectively differed in the quality of their various methodologies and analytical designs.

Abbreviations: BMF, breast milk fortifiers; EBM, expressed breast milk; NPN, non-protein nitrogen; TN, total nitrogen; PER, protein:energy ratio; PN, protein nitrogen.

* Corresponding author: G. McLeod, email gemma.mcleod@health.wa.gov.au 
Bedside milk analysis with the potential for targeted fortification is under development, with a recent study evaluating the accuracy of using IR analysis for small aliquots of milk, clinically relevant due to the smaller volumes of milk sometimes expressed by preterm mothers ${ }^{(25)}$. Fusch et al. ${ }^{(25)}$ highlighted that the near- and mid-IR spectroscopy equipment had variable accuracy when calibrated against micro analyses methods $(0.2 \mathrm{~g} / 100 \mathrm{ml}$ for protein, $0.5 \mathrm{~g} / 100 \mathrm{ml}$ for lipid and inaccurate measurements of lactose), which can be significant when feeding at low volumes. These values were similarly found in another study reviewing mid-IR spectroscopy, with lactose again being overestimated $^{(26)}$. A recent meta-analysis reported the mean values for each macronutrient in preterm and term milk from healthy mothers during the first 12 weeks of lactation, based on all available data, independent of the analysis used ${ }^{(27)}$. Inclusion criteria stipulated 24-h milk collections for fat and energy only. To account for study differences in the estimation of protein in breast milk (either including or excluding non-protein nitrogen (NPN), the authors conducted two meta-analyses of protein using the available data on each: an estimate of protein based on the assumption that all of the $\mathrm{N}$ is protein and a true protein estimate that excludes NPN. Two important findings from this meta-analysis were that milk content is relatively stable between weeks 2 and 12 of lactation and also that the most common difference reported between the quantity of total nitrogen (TN) and true protein estimates was $0.3 \mathrm{~g} / 100 \mathrm{ml}^{(27)}$. Although this difference appears to be small, it is likely to be clinically important, given that it represents about $30 \%$ of the amount of protein currently added by fortifiers to increase the protein content of preterm milk to ensure adequate growth.

The aims of this systematic literature review were to document the published values of preterm human milk composition over representative 24-h samples, assess the validity of the methodology in each of the studies, suggest preferred reference values for fortification based on this assessment and to determine the adequacy of industry-directed routine fortification in meeting nutrition targets.

\section{Methods}

Ovid Medline, Scopus, CINAHL and the Cochrane Library were searched using the strategy outlined in Fig. 1, limited to English language and publications from 1959 to 2013. Grey literature was identified through Trove limited to theses, ProQuest Dissertation and Theses and OAIster using the same search string.

The article review and exclusion processes are outlined in Fig. 2. In summary, all retrieved articles ( $n$ 7731) were reviewed by title and abstract, excluding articles identified as duplicates, non-English or not relevant to the study aim (Stage 2, Fig. 2). This was achieved by distributing all articles among the first four authors, who independently assessed the articles, conferring only when there was ambiguity about exclusion criteria. The remaining articles were reviewed at Stage 3 (Fig. 2) by at least two authors, and further exclusions were made if full texts could not be sourced, or if studies did not analyse preterm human milk for protein, energy, lactose, carbohydrate or lipid contents in representative 24-h samples. A 24-h sampling

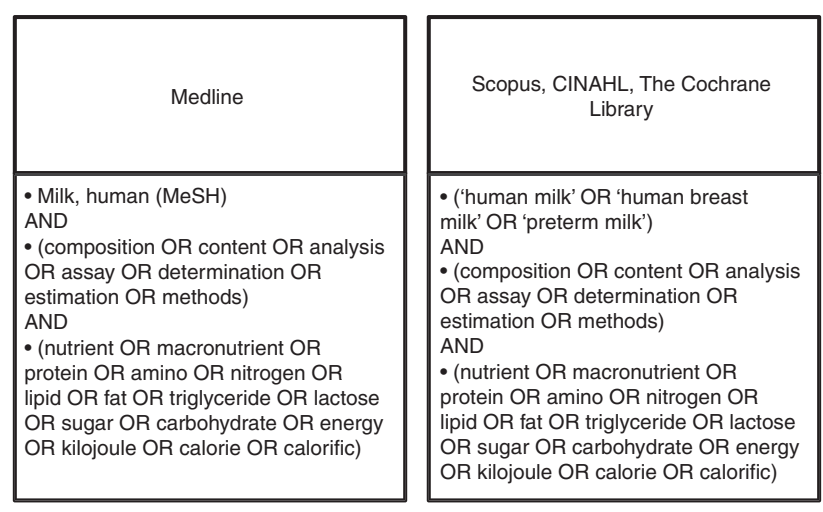

Fig. 1. Search strategies.

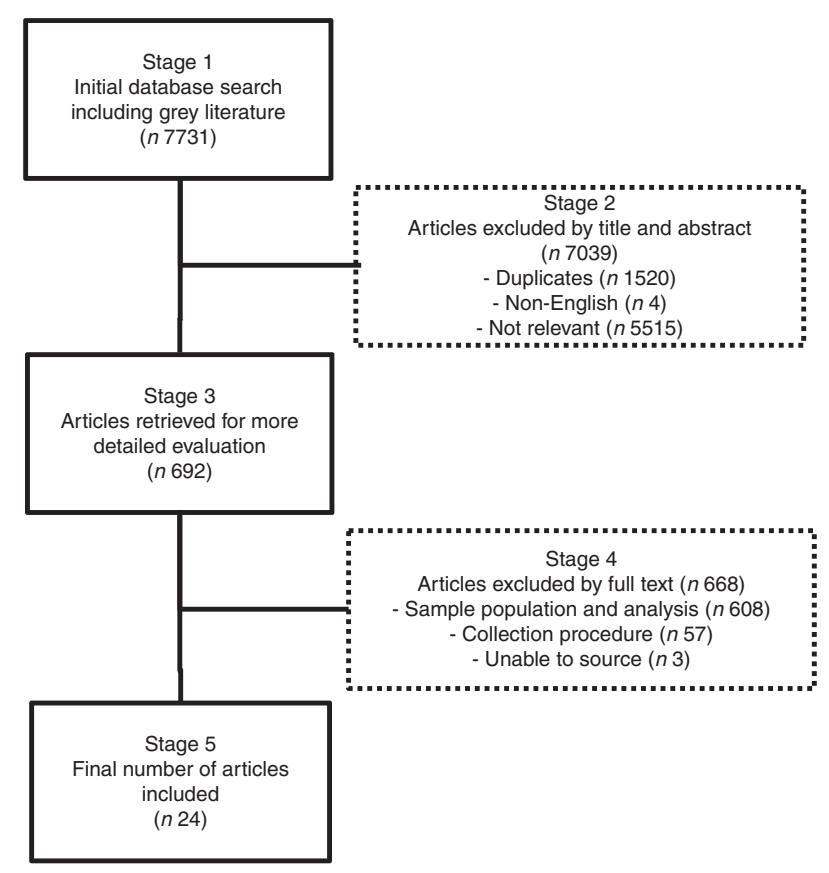

Fig. 2. Article review and exclusion process.

procedure was deemed necessary in order to obtain representative samples that accounted for diurnal and inter-feed variation in composition. We included 24-h milk expressions or representative samples comprising fore- and hind-milk samples collected in equal volumes from each expression throughout the day. Articles were also critiqued according to sample size, number of milk samples analysed and maternal demographics.

The included articles were examined with the aid of a hierarchical ranking of analytical methods (Fig. 3) based on the EN ISO/IEC 17025:2005 methodology practice, which considers methods according to selectivity, measurement uncertainty, repeatability/reproducibility and robustness within the matrix ${ }^{(28)}$. A method ranking system was not necessary for energy as the primary methods of determination were consistent within the articles assessed. Analyses expressing total carbohydrate and those only expressing the disaccharide lactose were considered separately.

All values were collated and standardised to $\mathrm{g} / 100 \mathrm{ml}$ for each macronutrient and $\mathrm{kJ}(\mathrm{kcal}) / 100 \mathrm{ml}$ for energy and were 

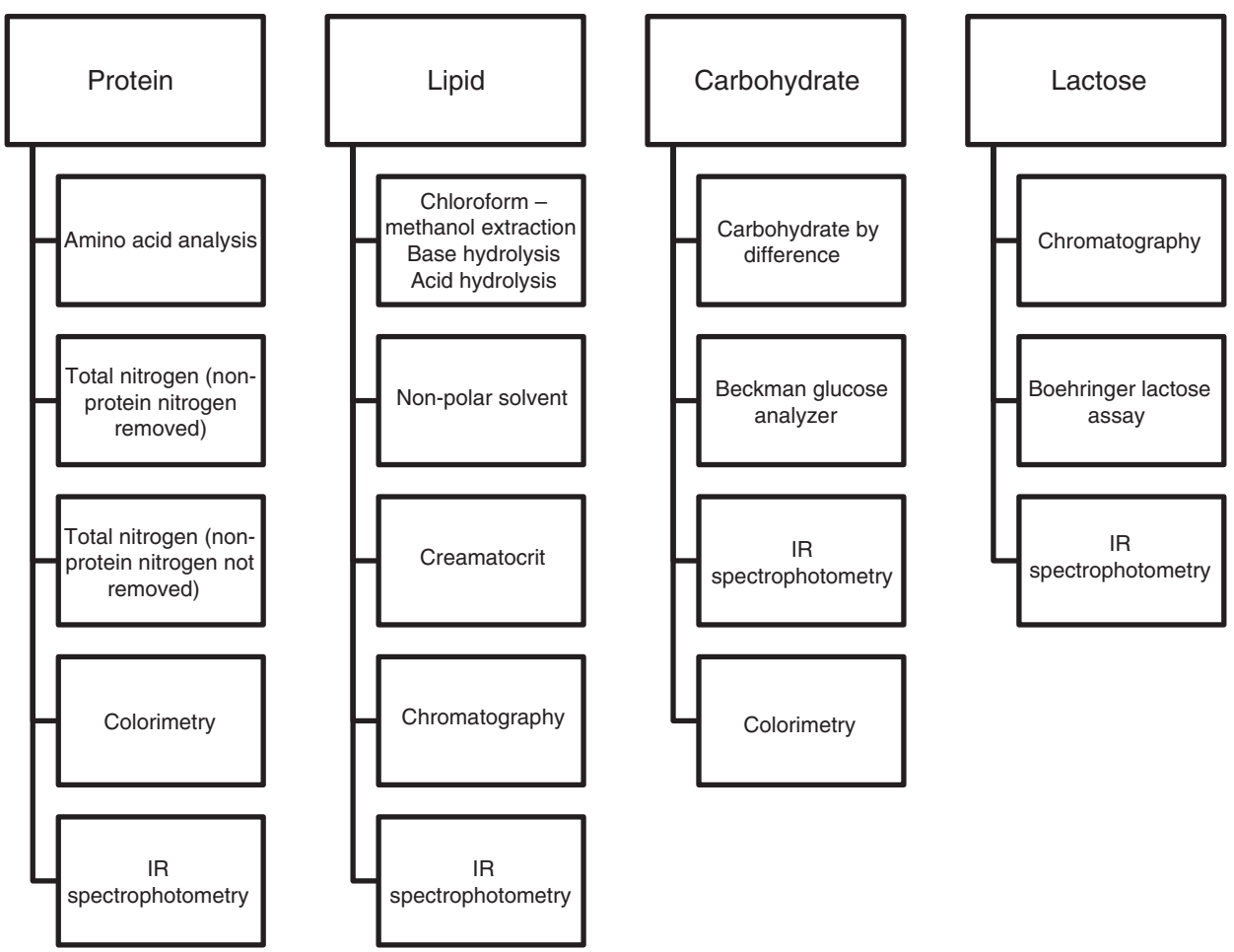

Fig. 3. Method ranking systems specific to protein, lipid, carbohydrate and lactose.

assigned to a lactation week according to the day of lactation (i.e. lactation week $1=$ inclusive of $1-7 \mathrm{~d}$, lactation week $2=$ inclusive of $8-14 \mathrm{~d}$, etc.) with values $>56 \mathrm{~d}$ compiled and reported as $\geq$ week 8 . Values that spanned multiple weeks were assigned to a week by the median day of the time period reported. In the case of values reported by type of milk, these were deemed to be of weeks 1,2 and 4 for colostrum, transitionary and mature milk, respectively.

Protein as determined by the Kjeldahl method results in determination of $\mathrm{TN}$, requiring adjustment for NPN and the subsequent conversion of this derived value to protein equivalents ${ }^{(29)}$. In this review, protein is defined as bioavailable $\mathrm{N}$ multiplied by the general conversion factor $6 \cdot 25^{(30)}$. Bioavailable $\mathrm{N}$ is protein nitrogen $(\mathrm{PN}=\mathrm{TN}-\mathrm{NPN})$ plus the proportion of NPN that is available for protein synthesis (approximately $27 \%$ of the NPN) ${ }^{(31)}$.

NPN values specific to different weeks of lactation (17.6\% for weeks $1-4$ of lactation ${ }^{(21)} ; 24 \%$ after week $4^{(31)}$ ) were used to standardise protein values across studies and to ensure that all values were representative of bioavailable proteinacious material. To achieve this, all reported values for PN were back calculated to TN. All TN values were then converted into bioavailable $\mathrm{N}$ using the applicable assumed NPN percentage (as above). In addition, where studies reported $\mathrm{PN}$ derived from the chemical determination of NPN, adjustment was made for bioavailable $\mathrm{N}$ where necessary. As there was little difference between the calculated and the chemically derived NPN component of milk, all studies using a form of the Kjeldahl method were used to suggest preferred mean protein values for weeks $1-8$ of lactation.

\section{Results}

In all, 7731 articles were reviewed by title and abstract, and 7039 articles were excluded as they were duplicates, nonEnglish or not relevant to the study aim (Stage 2, Fig. 2). Of the 692 articles reviewed at Stage 3 (Fig. 2), a further 668 articles were excluded because the investigators did not analyse preterm human milk for protein, energy, lactose, carbohydrate or lipid contents in representative 24-h samples or because full texts could not be sourced. The demographics of the included studies are reported in Table 1.

Results from the twenty-four studies included in this review are collated in Table 2 and depict mean and standardised reference ranges, organised by lactation week and macronutrients.

To determine a more accurate macronutrient composition, the means/medians (minimum, maximum) of the mean values reported in lactation week 1 and weeks $2-8$ by studies using the more robust methodology as per Fig. 3 are shown in Table 3. Protein values used contain bioavailable protein based on assumed NPN percentage. Energy values have been calculated from the mean values using the Atwater general factors ${ }^{(49)}$.

The mean and minimum, maximum values reported for lactation weeks 2-8 (Table 3) have been used to calculate incremental volume intakes $(\mathrm{ml} / \mathrm{kg}$ per $\mathrm{d})$ of breast milk that has been routinely fortified with human milk fortifier (Nutriprem, Cow and Gate; Nutricia). The fortifier provides an additional $1.1 \mathrm{~g}$ of protein, $2.8 \mathrm{~g}$ of carbohydrate and $67 \mathrm{~kJ}$ (16 kcal) of energy when added to $100 \mathrm{ml}$ of expressed breast milk (EBM) (Fig. 4 (a-e)). Table 4 illustrates the change to protein:energy ratio (PER) and quantifies protein intakes at incremental volumes 
Table 1. Studies included in the systematic review

\begin{tabular}{|c|c|c|c|c|}
\hline References & $\begin{array}{l}\text { Gestational age } \\
\text { (weeks) }\end{array}$ & Subjects & Lactation period & Measured content \\
\hline Anderson et al. ${ }^{(18)}$ & $26-33$ & 17 & Days $3-5,8-11,15-18,26-29$ & $\mathrm{~N}$, lipid, lactose, $\mathrm{E}^{\star}$ \\
\hline Anderson et al. ${ }^{(17)}$ & 28-36 & 14 & Days $3,7,14$ & $\mathrm{~N}$, lipid, carbohydrate, $\mathrm{E}$ \\
\hline Atkinson et al. ${ }^{(32)}$ & $26-33$ & 7 & Days 2-29 & $\mathrm{N}$ \\
\hline Atkinson et al. ${ }^{(21)}$ & $26-33$ & 7 & Days 2-29 & $\mathrm{N}$ \\
\hline Atkinson et al. ${ }^{(33)}$ & Average 28.3 & - & Days $6-8,13-15$ & $\mathrm{~N}$, lipid, lactose, $\mathrm{E}^{*}$ \\
\hline Bauer \& Gerss ${ }^{(6)}$ & $23-33$ & 102 & $1-8$ weeks & Pro, lipid, carbohydrate, E \\
\hline Beijers et al. ${ }^{(34)}$ & $25 \cdot 7-36$ & 45 & 1,2 and 4 weeks & $\mathrm{N}$ \\
\hline Beijers \& Schaafsma ${ }^{(35)}$ & $<36$ & 65 & Days $0-5,6-14,15-55$ & Lipid \\
\hline Chessex et al. ${ }^{(36)}$ & Average 30.3 & 11 & - & $\mathrm{N}$, lipid, carbohydrate, $\mathrm{E}^{*}$ \\
\hline Corvaglia et al. ${ }^{(13)}$ & $26-32$ & 55 & Day 10 & $\mathrm{~N}$, lipid \\
\hline Ehrenkranz et al. ${ }^{(37)}$ & $26-33$ & 21 & Days $2,7,14,28,42$ & Lipid \\
\hline Faerk et al. ${ }^{(38)}$ & $<32$ (average 28) & 101 & $1-10$ weeks & $\mathrm{N}$, lipid, carbohydrate, $\mathrm{E}^{*}$ \\
\hline Gross et al. ${ }^{(20)}$ & $27-32$ & 12 & Days $4,7,14,21,28$ & Pro, lipid, lactose, $E^{\star}$ \\
\hline Guerrini et al. ${ }^{(39)}$ & $29-37$ & 25 & Days $2,3,4,5,6,10,30$ & Lipid \\
\hline Jitta et al. ${ }^{(40)}$ & $26-33$ & 37 & Days $3-5,8-11,15-18,22-25$ & Pro, lipid, lactose, $E^{\star}$ \\
\hline Lemons et al. ${ }^{(16)}$ & $27-37$ & 20 & $\begin{array}{l}\text { Days } 7,14,21,28 \text { (then biweekly until } \\
\quad 44 \text { weeks postconceptual age) }\end{array}$ & $\mathrm{N}$, lipid, lactose, $\mathrm{E}$ \\
\hline Lemons et al. ${ }^{(41)}$ & $27-37$ & 20 & $\begin{array}{l}\text { Days } 7,14,21,28 \text { (then biweekly until } \\
\quad 44 \text { weeks postconceptual age) }\end{array}$ & $\mathrm{N}$ \\
\hline Lepage et al. ${ }^{(42)}$ & $26-36$ & 32 & Days $5-10,11-30$ & N, lipid, E \\
\hline Lucas \& Hudson ${ }^{(43)}$ & $26-36$ & 58 & Days 1-28 & $\mathrm{N}$ \\
\hline Maas et al. ${ }^{(44)}$ & $25-29$ & 79 & $1-11$ weeks & $\mathrm{N}$, lipid, lactose, carbohydrate, $\mathrm{E}^{*}$ \\
\hline Saarela et al. ${ }^{(45)}$ & Average 31.4 & 36 & 1 week, $1,2,3,4,5,6$ months & $\mathrm{N}$, lipid, lactose, $\mathrm{E}^{*}$ \\
\hline Sann et al. ${ }^{(46)}$ & $26-35$ & 41 & $<6,7-14,>15 d$ & Pro, lipid, lactose \\
\hline Silber et al. ${ }^{(47)}$ & Average 29.6 & 5 & Days 9-17 & $\mathrm{N}$, lipid, lactose, $\mathrm{E}$ \\
\hline Stevens $^{(48)}$ & 34-36 weeks & 7 & Days 3-25 & $\mathrm{N}$ \\
\hline
\end{tabular}

E, energy; *, calculated; Pro, protein.

when 0.5 and $1.0 \mathrm{~g}$ of protein powder (Beneprotein; Novartis) are added to $100 \mathrm{ml}$ of breast milk that has been routinely fortified.

\section{Discussion}

\section{Biological outcomes}

Individualising milk fortification on the basis of measured milk analysis to meet recommended intakes and growth targets is not always possible or pragmatic in the clinical setting. It requires consideration of the infant's clinical presentation, weight and prescribed feeding volume, is reliant on precise, accurate and expensive measuring equipment, and is labour intensive and time consuming. Instead, and commonly, fortifier is added to milk in routine amounts as directed by industry. The calculation and reporting of nutritional intakes is thus based on the formulation of the fortifier and an assumed milk composition. There are wide variations in milk composition between and within mothers and across the course of lactation, and there are many variants in the assumed milk composition used across neonatal centres to estimate intakes. Therefore, it is difficult to interpret studies that seek to investigate the role nutrition plays in clinical and growth outcomes in the neonatal setting, which ultimately leads to a paucity of good-quality, standardised data that can inform both nutritional practice and development of evidence-based nutritional guidelines.

In this review, we have reported the mean values and ranges of the macronutrient content of preterm breast milk per lactation week, using data from studies that used 24-h milk sampling (Table 2). This strategy was adopted to avoid making broad assumptions or oversimplifying data that are influenced by differences in study design and by the diurnal, within-feed and inter- and intra-maternal variations in milk composition. Selecting data from studies that have utilised the most robust methodology, we have suggested preferred reference mean values for the macronutrient composition of preterm colostrum and preterm mature breast milk (protein: 1.27; fat 3.46; carbohydrate $7 \cdot 34$; energy 66 ) for use in the clinical setting (Table 3). Global acceptance of these reference values may help standardise calculation and reporting of nutritional intakes and the development of evidence-based guidelines and sound nutritional practice. This will better direct clinicians, researchers and industry in the development of appropriate formulations of breast milk fortifiers (BMF) that address the wide variation in breast milk composition and guide the level of fortification required to better achieve preterm nutrition and growth targets.

Growth data of 13 years (2000-2013) collected from 362833 low birth weight infants (501-1500 g) in the Vermont Oxford Network ${ }^{(50)}$ have recently been published. These growth data were collected over the period when earlier and more aggressive nutrition regimens were being adopted and during a time when the highest-ever protein intakes were recommended for infants weighing $<1000 \mathrm{~g}^{(8,51)}$. It is concerning that as late as $2013,50 \%$ of low birth weight infants in this network were growth restricted at discharge $(<10$ th percentile), and $28 \%$ had severe growth failure $(<3 \text { rd percentile })^{(50)}$. This is despite the reformulation of several human milk fortifiers since the latest enteral guidelines were released in $2010^{(8)}$, suggesting that current nutrition regimens, including fortification practices, may still not be sufficiently optimised to meet the needs of extremely preterm infants. 


\section{N British Journal of Nutrition}

Table 2. Standardised protein, lipid, lactose/carbohydrate and energy values organised by lactation week (Standardised mean values and ranges)

\begin{tabular}{|c|c|c|c|c|c|c|c|c|c|c|c|c|c|c|c|}
\hline \multirow[b]{2}{*}{ Weeks } & \multirow[b]{2}{*}{ Author } & \multirow[b]{2}{*}{ Protein method based on } & \multicolumn{2}{|c|}{$\begin{array}{l}\text { Protein value } \\
(\mathrm{g} / 100 \mathrm{ml})\end{array}$} & \multirow[b]{2}{*}{ Lipid method based on } & \multicolumn{2}{|c|}{$\begin{array}{l}\text { Lipid value } \\
(\mathrm{g} / 100 \mathrm{ml})\end{array}$} & \multirow{2}{*}{$\begin{array}{l}\text { Lactose/carbohydrate } \\
\text { method based on }\end{array}$} & \multicolumn{2}{|c|}{$\begin{array}{c}\text { Lactose/carbohydrate value } \\
(\mathrm{g} / 100 \mathrm{ml})\end{array}$} & \multirow[b]{2}{*}{ Energy method based on } & \multicolumn{2}{|c|}{$\begin{array}{l}\text { Energy value } \\
(\mathrm{kJ} / 100 \mathrm{ml})\end{array}$} & \multicolumn{2}{|c|}{$\begin{array}{l}\text { Energy value } \\
(\mathrm{kcal} / 100 \mathrm{ml})\end{array}$} \\
\hline & & & Mean & Range & & Mean & Range & & Mean & Range & & Mean & Range & Mean & Range \\
\hline 1 & 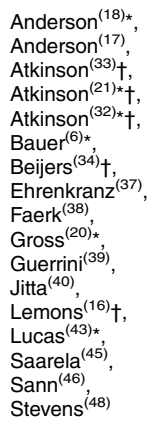 & 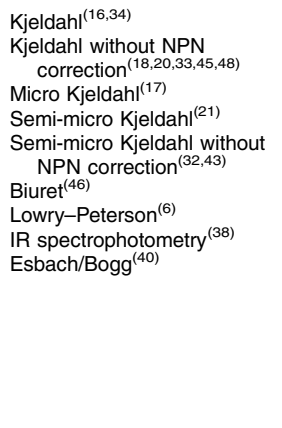 & 1.99 & $1.71-2.60$ & $\begin{array}{l}\text { Folch }^{(37,46)} \\
\text { Roese-Gottlieb } \\
\text { Colorimetry }^{(20,46,18,33)} \\
\text { De la Huerga } \\
\text { Van de Kamerg }^{(17)} \\
\text { Creamatocrit }^{(40)} \\
\text { IR spectrophotometry }^{(38)}\end{array}$ & $2 \cdot 80$ & $1 \cdot 60-3.90$ & $\begin{array}{l}\text { Lactose: } \\
\text { Chromatography }{ }^{(16,46)} \\
\text { Boehringer lactose assay } \\
\quad \text { kit }^{(18,33)} \\
\text { Unidentified } \\
\text { Unidentified } \\
\text { Unidentified } \\
\text { Total } \\
\text { Totarbohydrate: } \\
\text { Beckman Glucose Analyzer } \\
\text { IR spectrophotometry } \\
\text { I(17) }\end{array}$ & $\begin{array}{l}6.01 \\
6.73\end{array}$ & $\begin{array}{l}5 \cdot 04-7 \cdot 12 \\
6 \cdot 20-7 \cdot 10\end{array}$ & $\begin{array}{l}\text { Atwater specific factor } \\
\text { system } \\
\text { se) }^{(20)} \\
\text { Atwater general factor } \\
\text { system }^{(45)} \\
\text { Heats of combustion } \\
\text { factors }(18,33,38,40) \\
\text { Bomb calorimetry } \\
\text { (6,16,17) }\end{array}$ & 267.8 & $205 \cdot 0-309 \cdot 6$ & 64.0 & $49 \cdot 0-74 \cdot 0$ \\
\hline 2 & 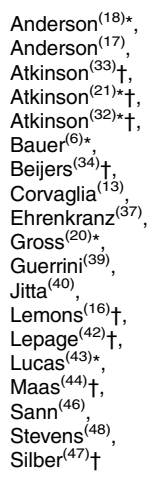 & 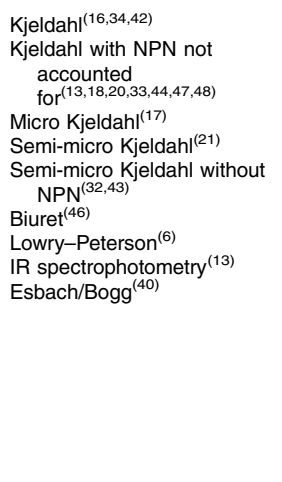 & 1.67 & $1 \cdot 46-2 \cdot 40$ & $\begin{array}{l}\text { Folch }^{(37,46)} \\
\text { Roese-Gottlieb } \\
\text { Colorimetry }^{(20,44), 18,33)} \\
\text { Gerber }^{(13)} \\
\text { De la Huerga } \\
\text { Jeejeebhoy } \\
\text { Van de Kamer) }^{(39)} \\
\text { Creamatocrit }^{(10)} \\
\text { IR spectrophotometry }^{(13)}\end{array}$ & 3.63 & $3 \cdot 49-4 \cdot 30$ & $\begin{array}{l}\text { Lactose: } \\
\text { Chromatography }{ }^{(16,46)} \\
\text { Boehringer lactose assay } \\
\quad \text { kit } \\
\text { Unidentified }^{(18,40)} \\
\text { Unidentified }^{(47)} \\
\text { Unidentified } \\
\text { Total carbohydrate: } \\
\text { Carbohydrate by difference } e^{(44)} \\
\text { Beckman Glucose Analyzer }\end{array}$ & $\begin{array}{l}5.94 \\
7.11\end{array}$ & $\begin{array}{r}5.31-6.86 \\
7.0-7.19\end{array}$ & $\begin{array}{l}\text { Atwater specific factor } \\
\text { system } \\
\text { sea) } \\
\text { Heats of combustion } \\
\text { factors }(18,33,40,44) \\
\text { Bomb calorimetry } \\
\text { (16,17,42,47) }\end{array}$ & $295 \cdot 8$ & $258.6-325.5$ & 70.7 & $61 \cdot 8-77 \cdot 8$ \\
\hline 3 & 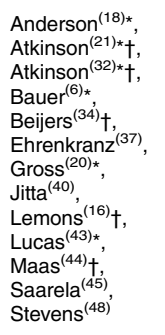 & 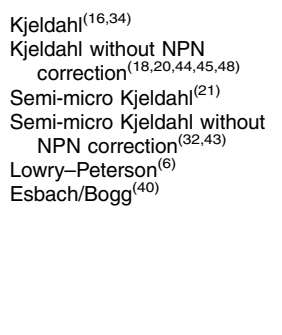 & 1.37 & $0.88-2 \cdot 10$ & $\begin{array}{l}\text { Folch }^{(37)} \\
\text { Roese-Gottlieb } \\
\text { Colorimetry }^{(16,18)} \\
\text { Creamatocrit }^{(6,40)}\end{array}$ & 3.84 & $3.24-4.80$ & $\begin{array}{l}\text { Lactose: } \\
\text { Chromatography } \\
\text { Boehringer lactose assay }^{(16)} \\
\text { kit }^{(18,44)} \\
\text { Unidentified }^{(40)} \\
\text { Unidentified }^{(45)} \\
\text { Unidentified } \\
\text { Total carbohydrate: }^{(20)} \\
\text { Carbohydrate by difference } \\
\text { Colorimetry }^{(4)}\end{array}$ & $\begin{array}{l}6.07 \\
7.42\end{array}$ & $\begin{array}{l}5.76-7.50 \\
7.25-7.60\end{array}$ & $\begin{array}{l}\text { Atwater specific factor } \\
\text { system } \\
\text { Atwater general factor } \\
\text { system } \\
\text { syst } \\
\text { Heats of combustion } \\
\text { factors } \\
\text { Bomb calorimetry } \\
\text { (16,40) }\end{array}$ & 297.9 & $252 \cdot 3-323 \cdot 8$ & $71 \cdot 2$ & $60 \cdot 3-77 \cdot 4$ \\
\hline 4 & $\begin{array}{l}\text { Bauer }(6) \star \\
\text { Faerk }^{(38)} \\
\text { Guerrini } \\
\text { Lemons }^{(39)} \\
\text { Maass }^{(44)} \dagger\end{array}$ & $\begin{array}{l}\text { Kjeldahl( }{ }^{(41)} \\
\text { Kjeldahl without NPN } \\
\text { correction }^{(44)} \\
\text { Lowry-Peterson }^{(6)} \\
\text { IR spectrophotometry }\end{array}$ & 1.33 & $0.93-2.00$ & $\begin{array}{l}\text { Roese-Gottlieb }{ }^{(44)} \\
\text { De la Huerga } \\
\text { IR spectrophotometry }^{(38)}\end{array}$ & 3.88 & $3.50-4.60$ & $\begin{array}{l}\text { Lactose: } \\
\text { Boehringer lactose assay kit }{ }^{(44)} \\
\text { Total carbohydrate: } \\
\text { Carbohydrate by difference }^{(44)} \\
\text { IR spectrophotometry } \\
\text { (38) }\end{array}$ & $\begin{array}{l}6.02 \\
7.47\end{array}$ & $7.40-7.53$ & $\begin{array}{l}\text { Heats of combustion } \\
\text { factors }^{(38,44)}\end{array}$ & 312.5 & $294.5-330.5$ & 74.7 & $70 \cdot 4-79 \cdot 0$ \\
\hline 5 & $\begin{array}{l}\text { Bauer }{ }^{(6) *}, \\
\text { Ehrenkranz } \\
\text { Lemons } s^{(16)} \dagger \\
\operatorname{Maas}^{(44)} \dagger\end{array}$ & $\begin{array}{l}\text { Kjeldahl(16) } \\
\text { Kjeldahl without NPN } \\
\text { correction }^{(44)} \\
\text { Lowry-Peterson }^{(6)}\end{array}$ & 1.46 & $1.23-1.90$ & $\begin{array}{l}\text { Folch }^{(37)} \\
\text { Roese-Gottlieb } \\
\text { Colorimetry }{ }^{(46)}\end{array}$ & 3.63 & $3 \cdot 17-4 \cdot 36$ & $\begin{array}{l}\text { Lactose: } \\
\text { Chromatography }{ }^{(16)} \\
\text { Boehringer lactose assay kit }{ }^{(44)} \\
\text { Total carbohydrate: } \\
\text { Carbohydrate by difference }^{(44)}\end{array}$ & $\begin{array}{l}6.32 \\
7.25\end{array}$ & $\begin{array}{l}5.71-7.21 \\
7.04-7.45\end{array}$ & $\begin{array}{l}\text { Heats of combustion factors }{ }^{(44)} \\
\text { Bomb calorimetry }\end{array}$ & 286.6 & $274 \cdot 9-293 \cdot 3$ & 68.5 & $65 \cdot 7-70 \cdot 1$ \\
\hline
\end{tabular}




\section{NS British Journal of Nutrition}

\section{Table 2. Continued}

\begin{tabular}{|c|c|c|c|c|c|c|c|c|c|c|c|c|c|c|c|}
\hline \multirow[b]{2}{*}{ Weeks } & \multirow[b]{2}{*}{ Author } & \multirow[b]{2}{*}{ Protein method based on } & \multicolumn{2}{|c|}{$\begin{array}{l}\text { Protein value } \\
(\mathrm{g} / 100 \mathrm{ml})\end{array}$} & \multirow[b]{2}{*}{ Lipid method based on } & \multicolumn{2}{|c|}{$\begin{array}{l}\text { Lipid value } \\
\text { (g/100 ml) }\end{array}$} & \multirow{2}{*}{$\begin{array}{l}\text { Lactose/carbohydrate } \\
\text { method based on }\end{array}$} & \multicolumn{2}{|c|}{$\begin{array}{c}\text { Lactose/carbohydrate value } \\
(\mathrm{g} / 100 \mathrm{ml})\end{array}$} & \multirow[b]{2}{*}{ Energy method based on } & \multicolumn{2}{|c|}{$\begin{array}{l}\text { Energy value } \\
(\mathrm{kJ} / 100 \mathrm{ml})\end{array}$} & \multicolumn{2}{|c|}{$\begin{array}{l}\text { Energy value } \\
(\mathrm{kcal} / 100 \mathrm{ml})\end{array}$} \\
\hline & & & Mean & Range & & Mean & Range & & Mean & Range & & Mean & Range & Mean & Range \\
\hline 6 & $\begin{array}{l}\text { Bauer(6)* } \\
\text { Faerkr(38)' }^{(38)} \\
\text { Maas }^{(44)} \dagger\end{array}$ & $\begin{array}{l}\text { Kjeldahl without NPN } \\
\quad \text { correction( } \\
\text { Low) } \\
\text { Lowry-Peterson } \\
\text { IR spectrophotometry }\end{array}$ & 1.38 & $1.05-1.90$ & $\begin{array}{l}\text { Roese-Gottlieb } \\
\text { IR spectrophotometry }\end{array}$ & $4 \cdot 24$ & 3.57-4.90 & $\begin{array}{l}\text { Lactose: } \\
\text { Boehringer lactose assay kit }{ }^{(44)} \\
\text { Total carbohydrate: } \\
\text { Carbohydrate by difference } \\
\text { IR spectrophotometry } \\
\text { I }{ }^{(48)}\end{array}$ & $\begin{array}{l}6 \cdot 18 \\
7 \cdot 40\end{array}$ & $7 \cdot 30-7 \cdot 50$ & $\begin{array}{l}\text { Heats of combustion } \\
\text { factors(38,44) } \\
\text { Bomb calorimetry }\end{array}$ & 331.4 & $293 \cdot 3-362 \cdot 3$ & $79 \cdot 2$ & $70 \cdot 1-86 \cdot 6$ \\
\hline 7 & $\begin{array}{l}\text { Bauer }^{(6) *}, \\
\text { Faerk }^{(38)} \\
\text { Lemons }^{(16)} \dagger \\
\left.\text { Maass }^{(44)}\right) \\
\text { Saarela }^{(45)}\end{array}$ & $\begin{array}{l}\text { Kjeldahl }^{(16)} \\
\text { Kjeldahl without NPN } \\
\text { correction }^{(44,45)} \\
\text { Lowry-Peterson }^{(6)} \\
\text { IR spectrophotometry }^{(38)}\end{array}$ & 1.31 & $1.03-1.80$ & $\begin{array}{l}\text { Roese-Gottlieb } \\
\text { Colorimetry(146) } \\
\quad \text { IR spectrophotometry }\end{array}$ & 3.92 & $2 \cdot 94-4 \cdot 80 \ddagger$ & $\begin{array}{l}\text { Lactose: } \\
\text { Chromatography }{ }^{(16)} \\
\text { Boehringer lactose assay kit }{ }^{(44)} \\
\text { Unidentifieded(45) } \\
\text { Total carbohydrate: } \\
\text { Carbohydrate by difference }{ }^{(44)} \\
\text { IR spectrophotometry }{ }^{(48)}\end{array}$ & $\begin{array}{l}6.83 \\
7.29\end{array}$ & $\begin{array}{l}5 \cdot 95-7 \cdot 45 \ddagger \\
7 \cdot 27-7 \cdot 30\end{array}$ & $\begin{array}{l}\text { Atwater general factor } \\
\text { system } \\
\text { Heats of combustion } \\
\text { factors }{ }^{(38,44)} \\
\text { Bomb calorimetry }{ }^{(16)}\end{array}$ & 290.8 & $263 \cdot 6-334 \cdot 7$ & 69.5 & $63 \cdot 0-80.0 \ddagger$ \\
\hline$\geq 8$ & 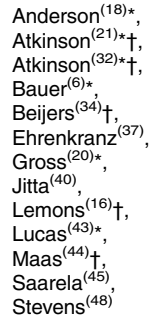 & 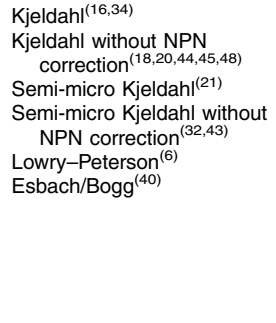 & 1.37 & $0 \cdot 88-2 \cdot 10$ & $\begin{array}{l}\text { Folch }^{(37)} \\
\text { Roese-Gottlieie(20,44,45) } \\
\text { Colorimetryy } \\
\text { Creamatocrit } \\
\text { Cr,40) }\end{array}$ & 3.84 & $3 \cdot 24-4 \cdot 80$ & $\begin{array}{l}\text { Lactose: } \\
\text { Chromatography }{ }^{(16)} \\
\text { Boehringer lactose assay } \text { kit }^{(18,44)} \\
\text { Unidentified }^{(40)} \\
\text { Unidentified }^{(45)} \\
\text { Unidentified } \\
\text { Total carbohydrate: } \\
\text { Carbohydrate by difference }^{(24)} \\
\text { Colorimetry } \\
\left({ }^{(6)}\right.\end{array}$ & $\begin{array}{l}6.07 \\
7.42\end{array}$ & $\begin{array}{l}5.76-7.50 \\
7 \cdot 25-7.60\end{array}$ & $\begin{array}{l}\text { Atwater specific factor } \\
\text { system } \\
\text { Atwater general factor } \\
\text { system } \\
\text { Heats of combustion } \\
\text { factors }(18,40,44) \\
\text { Bomb calorimetry } \\
\text { (6,16) }\end{array}$ & 297.9 & $252 \cdot 3-323 \cdot 8$ & $71 \cdot 2$ & $60 \cdot 3-77 \cdot 4$ \\
\hline
\end{tabular}

NPN, non-protein nitrogen.

.

t Articles in which protein values were calculated from reported $\mathrm{N}$ values, using the conversion factor 6.25 .

Ranges inclusive of values obtained up to 16 weeks of lactation. 
Table 3. Macronutrient composition of lactation week 1 and lactation weeks $2-8$ using systematically selected data (Means/medians of values reported for Lactation week 1; means/medians (minimum and maximum) of values reported for each of Weeks 2-8)

\begin{tabular}{|c|c|c|c|c|c|}
\hline & Protein $(\mathrm{g} / 100 \mathrm{ml})$ & Lipid (g/100 ml) & $\begin{array}{l}\text { Carbohydrate } \\
\text { (lactose, g/100 ml) }\end{array}$ & $\begin{array}{l}\text { Calculated energy } \\
(\mathrm{kJ} / 100 \mathrm{ml})\end{array}$ & $\begin{array}{l}\text { Calculated energy } \\
\text { (kcal/100 ml) }\end{array}$ \\
\hline Lactation week 1 & $1 \cdot 90 / 1 \cdot 88^{(16-18,20,21,32-34,43,45,48)}$ & $2 \cdot 59 / 2 \cdot 63^{(20,37,45,46)}$ & $\begin{array}{l}6 \cdot 55 / 6 \cdot 55^{(18)} \\
5 \cdot 66 / 5 \cdot 61^{(16,18,33,46)}\end{array}$ & 238.95 & $57 \cdot 11$ \\
\hline Lactation weeks 2-8 & $\begin{array}{l}1 \cdot 27 / 1 \cdot 24 \\
(1 \cdot 02-1 \cdot 58)^{(13,16-18,20,21,32-34,36,41-45,47,48)}\end{array}$ & $\begin{array}{l}3 \cdot 46 / 3 \cdot 54 \\
(3 \cdot 25-3 \cdot 69)^{(20,35,37,44-46)}\end{array}$ & $\begin{array}{l}7 \cdot 34 / 7 \cdot 28(7 \cdot 11-7 \cdot 53)^{(18,44)} \\
6 \cdot 15 / 6 \cdot 04 \\
(5 \cdot 93-6 \cdot 32)^{(16,18,33,44,46)}\end{array}$ & $\begin{array}{l}274 \cdot 5 / 274 \cdot 9 \\
(264 \cdot 72-281 \cdot 04)\end{array}$ & $\begin{array}{l}65 \cdot 6 / 65 \cdot 7 \\
(63 \cdot 27-67 \cdot 17)\end{array}$ \\
\hline
\end{tabular}

Commercial BMF currently provide between 1.0 and $1.1 \mathrm{~g}$ protein/100 $\mathrm{ml}$ EBM. Polberger et al. ${ }^{(52)}$ used a BMF containing $1 \mathrm{~g}$ protein $/ 100 \mathrm{ml}$ EBM to routinely fortify milk and calculated protein intakes of $3.05 \mathrm{~g} / \mathrm{kg}$ per $\mathrm{d}$ in the control arm of the trial, using milk composition data measured with IR technology. Similarly, McLeod et al. ${ }^{(53)}$ used a BMF containing $1 \mathrm{~g}$ protein/ $100 \mathrm{ml} \mathrm{EBM}$ and additional protein powder up to $0.5 \mathrm{~g} / 100 \mathrm{ml}$ EBM to fortify the milk of preterm infants in the control arm of a randomised trial. Mean volume and protein intakes of $153 \mathrm{ml} / \mathrm{kg}$ per $\mathrm{d}$ and $3.9 \mathrm{~g} / \mathrm{kg}$ per $\mathrm{d}$, respectively, were calculated for these infants during the fortification period, using milk composition data obtained with mid-IR technology. Miller et al. ${ }^{(54)}$ has also shown improved protein intakes of $4.2 \mathrm{~g} / \mathrm{kg}$ per $\mathrm{d}$ in the first 4 weeks of a study of preterm infants born $<31$ weeks of gestation who were fed a trial-based BMF containing $1.4 \mathrm{~g}$ of protein $/ 100 \mathrm{ml}$ of breast milk $(160,139-170 \mathrm{ml} / \mathrm{kg}$ per d) compared with controls who were fed an isoenergetic BMF containing $1 \mathrm{~g}$ protein to achieve intakes of $3.6 \mathrm{~g} / \mathrm{kg}$ per $\mathrm{d}$ (164, 149-171 ml/kg per d).

The American Academy of Pediatrics has stipulated that breast milk should be the primary diet of all preterm infants and that it should be appropriately fortified for those with birth weights $<1500 \mathrm{~g}$ to target intra-uterine growth rates ${ }^{(5)}$. Using our preferred minimum and mean values for preterm milk (Table 3) and by using fortification levels as directed by industry, we have demonstrated (Fig. 4(c)) that the recommended protein targets of $4.0-4.5 \mathrm{~g} / \mathrm{kg}$ per $\mathrm{d}$ considered necessary for the growth of preterm infants weighing $<1000 \mathrm{~g}^{(8)}$ may not be achieved below volumes of 190-210 and $170-190 \mathrm{ml} / \mathrm{kg}$ per $\mathrm{d}$, respectively. This is concerning, given that lower volumes may be prescribed for extremely preterm infants to minimise risk of long-term morbidity such as chronic lung disease and patent ductus arteriosus ${ }^{(8)}$. It is also concerning that the PER of fortified milk, calculated using these preferred values, does not appear to meet recommendations for these infants (Fig. 4(e)). From our estimations, it seems likely that this is due to the insufficient protein and high carbohydrate content of fortifiers, the latter adding a substantial energy load to the final composition of fortified milk, resulting in excessive energy intakes at reasonably low volumes (Fig. 4(d)) and carbohydrate intakes exceeding recommendations beyond volumes of $140 \mathrm{ml} / \mathrm{kg}$ per d (Fig. 4(b)).

Table 4 depicts simulated fortified intakes achieved at incremental fluid intakes, based on our preferred mean values, and a commercially available BMF $(1.1 \mathrm{~g}$ protein $/ 100 \mathrm{ml}$ EBM $)$ and 0.5 or $1.0 \mathrm{~g}$ of protein supplement $(0.86 \mathrm{~g}$ protein/g protein powder). Notably, protein, energy and PER targets for infants weighing $<1000 \mathrm{~g}$ are achieved when $0.5 \mathrm{~g}$ of protein powder is added to feeds in addition to BMF and fed at volumes between 140 and 160, and similarly these targets are achieved at lower volumes (130-140 ml/kg per d) when $1.0 \mathrm{~g}$ of protein powder is added in addition to routine amounts of BMF. This level of fortification is currently being practised in some neonatal units around the globe, with the understanding that additional, intact protein powder does not adversely affect osmolality of the feeds ${ }^{(55)}$, and with acceptance that protein intakes may be greater than needed for some preterm infants if the native content of the milk is actually higher than anticipated $^{(56)}$. The efficacy and safety of these fortification practices have not been well studied, and further research is needed to determine the best formulation and the amount of fortifier that can be safely added to preterm milk to optimise preterm growth, developmental and metabolic outcomes. It seems increasingly clear that one size may not fit all; different strength fortifiers may be required to meet the needs of very low weight or poorly growing infants and to address the variations in milk composition between mothers and across the course of lactation.

\section{Justification for the preferred reference values of preterm milk}

Protein. Amino acid analysis is the most accurate method for determining true protein content ${ }^{(22)}$; however, this method is time consuming and costly. ${ }^{(22)}$ The Kjeldahl method is the most accurate indirect determination of protein content ${ }^{(57,58)}$, and it - or its more sensitive derivations ${ }^{(59)}$ (micro and semi-micro Kjeldahl) - is the most commonly used method employed by studies in this review ${ }^{(13,16-18,20,21,32-34,42-45,47,48)}$. The Kjeldahl method involves liberation of TN from a sample ${ }^{(60)}$. A second analysis improves the accuracy by determining NPN and subtracting this from $\mathrm{TN}$ to determine $\mathrm{PN}^{(60)}$. During protein precipitation, peptides remain in the supernatant and are attributed to NPN, resulting in a minor underestimation of true protein as they are excluded from the derived $\mathrm{PN}^{(60)}$; four studies $^{(16,17,21,34)}$ reported chemically determined NPN values, which were used to determine bioavailable protein and were compared with the bioavailable protein using an assumed NPN percentage. Differences in bioavailable protein calculated using chemically derived NPN compared with using assumed values during week 1 to week 4 of lactation ranged from -0.06 to $0 \cdot 21 \mathrm{~g}$. Although it is technically superior to chemically derived NPN, the small differences between bioavailable proteins using the above methods are reassuring, and thus all derivations of Kjeldahl with NPN correction applied were considered to be superior. 
(a)

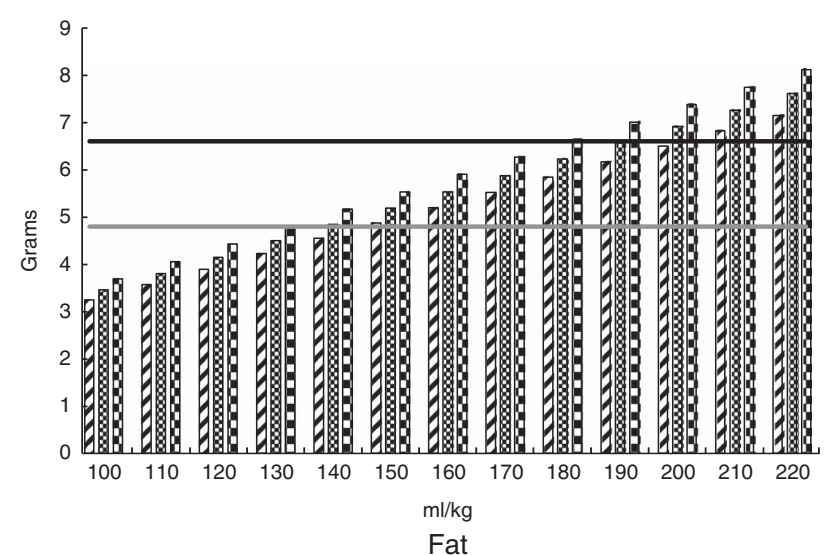

(c)

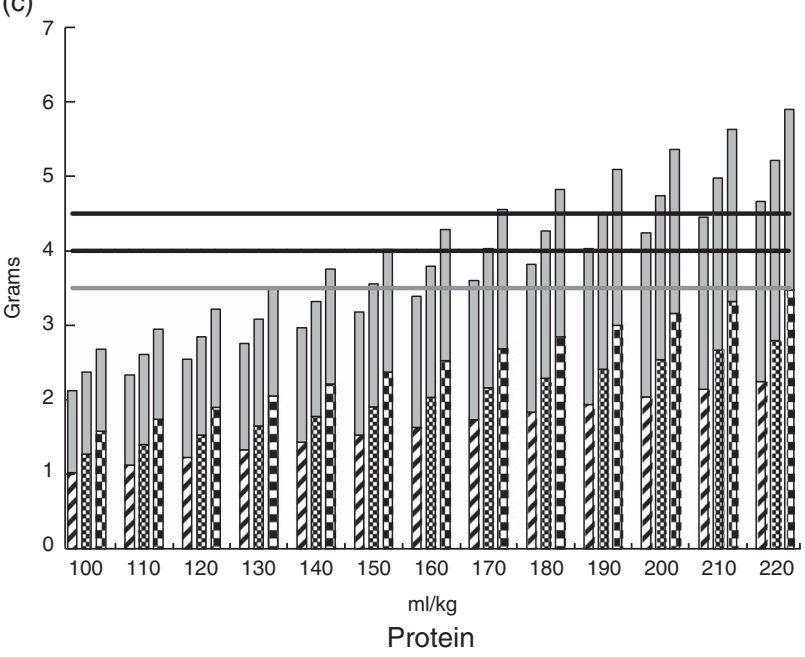

(b)

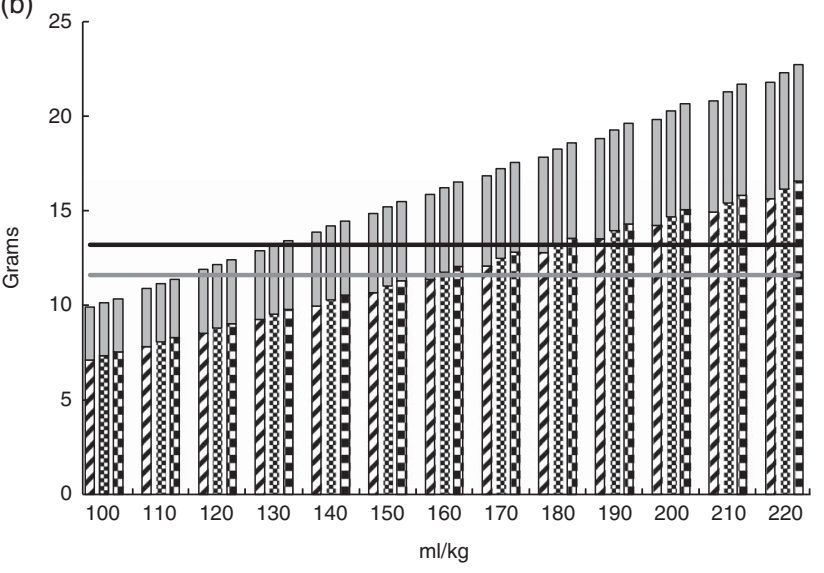

Carbohydrate

(d)

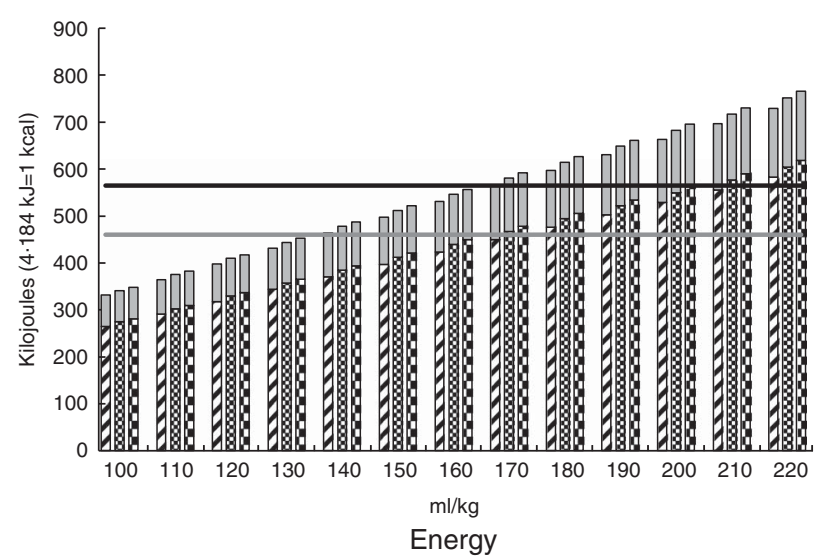

(e)

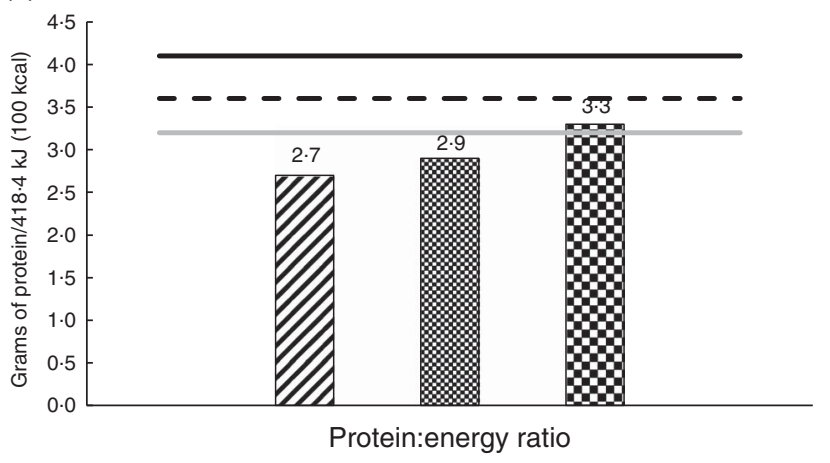

Fig. 4. Estimated intakes at incremental volumes and protein:energy feed ratios derived from routinely fortified milk (4.4 $\mathrm{g}$ Nutriprem/100 $\mathrm{ml}$ breast milk), using the minimum, mean and maximum values for milk components at lactation weeks 2-8 as described in Table 3. (a) Fat; (b) carbohydrate; (c) protein; (d) energy and (e) protein:energy ratio. The fortifier does not contain fat. 四, minimum value for component in breast milk; 圆, mean value for component in breast milk; $\mathbb{B}$, maximum value for component in breast milk; $\square$, fortifier; —_ minimum recommended daily intake; $\_$, minimum recommended daily intake $(<1000 \mathrm{~g})$, maximum recommended daily intake $(1000-1800 \mathrm{~g})$; _ _ maximum recommended daily intake.

The modified Lowry-Petersen and Biuret assays use colorimetry to quantify protein concentration ${ }^{(60)}$. The Biuret assay, used by Sann et $a l .{ }^{(46)}$, is limited in that it requires a large number of samples ${ }^{(61)}$, has low sensitivity with detection starting at $1 \mathrm{~g} / \mathrm{l}$ and can be confounded by other components in milk $^{(60)}$. Bauer \& Gerss ${ }^{(6)}$ used the Lowry-Petersen method to determine protein content in the milk of a large sample of mothers; this study reported the highest protein values for each lactation week. Reliability of the Lowry-Petersen assay is dependent on technique, which demands high accuracy in 


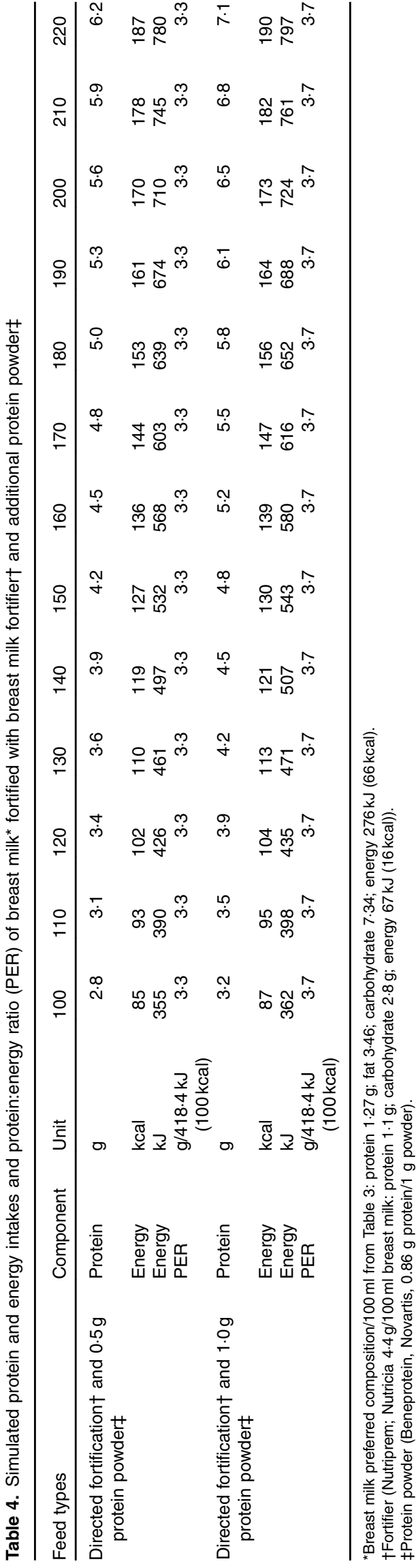

achieving a 100-fold dilution and precise timing when adding the reagent ${ }^{(60)}$.

Jitta et al. ${ }^{(40)}$ used a variation of the Esbach's method, which was designed to measure protein in urine ${ }^{(62)}$. This, together with the socio-cultural demographics of the Kenyan women in this study ${ }^{(40)}$, may account for the low protein values reported for weeks 3 and 4 compared with those determined by other methods.

In addition, two studies used IR spectrophotometry methods to determine $\mathrm{TN}$ and true protein concentrations, respectively $^{(13,38)}$. This indirect determination can vary in accuracy depending on the calibration of the individual machines ${ }^{(12)}$, the choice of the reference method used and the diversity and number of samples used in the calibration set ${ }^{(63)}$. That aside, it is noteworthy that the protein values reported in these studies were consistent with values found by more direct methods.

Lipid. During the first 5 weeks of lactation, lipid concentration appears to increase, with convergence of the range of reported values. Obtaining representative lipid samples requires 24-h collections to allow for variation as well as careful mixing of the sample at $38^{\circ} \mathrm{C}$ before removing an aliquot for analysis ${ }^{(23)}$. The preparation and storage of a sample is important to maintain its integrity; a limit of $14 \mathrm{~d}$ at $-20^{\circ} \mathrm{C}$ is recommended to prevent lipolysis ${ }^{(64)}$. Only one study specified the duration of storage at such temperatures ${ }^{(45)}$. This susceptibility to lipolysis can be overcome by storing samples at $-70^{\circ} \mathrm{C}$ or below ${ }^{(64)}$, a strategy used in three of the included studies ${ }^{(6,16,37)}$. Of note, this should be used by studies determining lipid concentration without using extraction techniques. Preferred methods for determining total lipid concentration include modified Folch and RoeseGottlieb methods, which both use chloroform-methanol extraction followed by gravimetric determination ${ }^{(64,65)}$. Insull \& Ahrens $^{(66)}$ compared modified Folch with Roese-Gottlieb methods, and found that the total lipids that were recovered were similar between the two methods but the Folch method recovered phospholipids more effectively ${ }^{(23)}$; four articles (Maas et $a l^{(44)}$, Beijers et $a l .{ }^{(35)}$, Saarela et $a l .{ }^{(45)}$ and Gross et $a l{ }^{(20)}$ ) used the Roese-Gottlieb method, and the narrow range for total lipid reported over the first 8 weeks of lactation in these studies emphasises the robust nature of their methodology. The Folch technique was used by Ehrenkranz et $a l .{ }^{(37)}$ and Sann et $a l .{ }^{(46)}$; however, the reported results using this method were only comparable for matched lactation weeks 1 and 2, in which differences in fat concentration of up to $1.35 \mathrm{~g} / 100 \mathrm{ml}$ were noted.

Chloroform-methanol extraction followed by colorimetric determination was used by Anderson et $a l^{\left({ }^{(18)}\right.}$ and Atkinson et $a l .{ }^{(33)}$. Lemons et $a l .{ }^{(16)}$ used colorimetric determination but used sulphuric acid for extraction ${ }^{(67)}$. The colorimetric determination of lipid yielded higher values for week 1 of lactation, compared with those determined by the RoeseGottlieb and Folch methods, but were similar for the remaining weeks. A modified De la Huerga method of extraction followed by photometric determination was used by Guerrini et al. ${ }^{(39)}$. This method was not as specific as previously mentioned methods, as there was no precipitation of protein before 
photometrically reading the sample's turbidity. The method used by Silber et $a l .{ }^{(47)}$ was crude fat determination as lipid extraction was performed using non-polar solvents, and therefore did not account for phospholipids. Anderson et al. ${ }^{(17)}$ calculated total lipid concentration on the basis of average fatty acid chain length, a method less robust than direct measurements.

The creamatocrit technique is a simple, rapid and inexpensive measure of fat in milk ${ }^{(64,68)}$. The method is limited by the subjectivity associated with reading the measurement, and the potential for overestimating the lipid content due to significant unpacking of the lipid column if the sample is read after $30 \min ^{(6,68)}$. However, the creamatocrit method has an accuracy of $\pm 10 \%$, which is considered by some to be adequate in most clinical and research settings ${ }^{(68)}$. Jitta et al. ${ }^{(40)}$ and Bauer \& Gerss $^{(6)}$ both used the creamatocrit technique, and their results were comparable with the Roese-Gottlieb method for weeks 1-3 but appeared to be an overestimation for week 4 . The Gerber method used by Corvaglia et al. ${ }^{(13)}$ uses an adequate extraction technique; however, the volumetric determination is inferior to the gravimetric methods and requires a large sample size ${ }^{(68)}$.

IR spectrophotometers have the ability to ascertain values for all macronutrients in one measurement; however, this is an indirect measurement and the machine must be calibrated against direct analysis ${ }^{(63)}$. The strength of the calibration depends on the reference chemistry used and the number and range of samples ${ }^{(63)}$. Homogenisation is an important step in preparing milk samples as it decreases the variability in fat globule size and subsequent light-scattering effect of larger globules, improving the accuracy of measurement ${ }^{(25)}$. Reducing the diameter of the fat globule to $<3 \mu \mathrm{m}$ can be achieved through either manual or ultrasonographic methods; however, utilising an ultrasound processor is associated with high measured values for $\mathrm{N}$, energy and particularly fat and lactose $^{(69)}$. Neither of the studies has reported whether the samples underwent homogenisation before analysis. Corvaglia et $a l .{ }^{(13)}$ used near-IR, with a detailed description of calibration. This study found a correlation of 0.808 between IR analysis and the Roese-Gottlieb method ${ }^{(13)}$. Faerk et $a{ }^{\left({ }^{(38)}\right.}$ used mid-IR spectrophotometry but did not provide information on the calibration of their machine, which is critical in establishing the reliability of the results ${ }^{(63)}$. This study consistently reported higher lipid concentrations than those reported by others using different methods.

Carbohydrate/lactose. Lactose is the predominant disaccharide carbohydrate in human milk (approximately $70-83 \%$ of total carbohydrates); however, human milk also contains free glucose and galactose, as well as numerous oligosaccharides ${ }^{(70)}$. Of the studies reported in Table 1, nine reported lactose concentrations $^{(16,18,20,33,40,44-47)}$ and five reported total carbohydrate $\mathrm{e}^{(6,17,36,38,44)}$. As expected, the total carbohydrate composition range was consistently higher than lactose. Further, both lactose and total carbohydrate followed the same trend, which was to increase gradually over weeks $1-4$ and remain relatively stable from then on. Although it may be preferable from a clinical perspective to identify a total carbohydrate value, the majority of studies with high methodological quality measured lactose; two reliable methodologies identified for lactose determination were used by five studies ${ }^{(16,18,33,44,46)}$. These methods were chromatography, with Lemons et al. ${ }^{(16)}$ using GLC and Sann et $a l^{(46)}$ using ion-exchange chromatography, as well as the Boehringer lactose assay used by Anderson et $a l^{(18)}$, Maas et $a l^{(44)}$ and Atkinson et $a l^{(33)}$. Although these methods differ, they are each primary methods of analysis, specific for lactose and are not confounded by carbohydrate interference. The chromatographic methods achieve this by virtue of their calibrated parameters being lactose specific, and the Boehringer lactose assay accounts for free glucose. There were four studies included in this review reporting lactose values in which the methodology was unable to be sufficiently identified ${ }^{(20,40,45,47)}$. It is also noteworthy that Saarela et al. ${ }^{(45)}$ used an unidentified enzymatic degradation method, which greatly increased the range reported in weeks 1 and 4 where values from their study were included. It is possible that this methodology may not have accounted for free glucose, and thus overestimated lactose; however, this is unlikely to be significant. Of the studies reporting total carbohydrate, the main limitation was the infrequency with which measurements were taken or reported over the weeks of lactation. Chessex et al. $^{(36)}$ and Bauer \& Gerss ${ }^{(6)}$ only contributed to the ranges reported in weeks 3 and 4, respectively. Although Faerk et $a l .{ }^{(38)}$ reported values for multiple weeks, it should be noted that the validity of the IR spectrophotometry values is not clearly elucidated because the calibration method was not explicitly stated. Despite the potential for confounding errors in a multistep process, Maas et $a l^{(44)}$ used sound methodology, calculating total carbohydrate by difference, and provided the greatest contribution to the carbohydrate trend, reporting values over weeks 2-8.

Energy. Bomb calorimetry accurately measures total energy content and was used in a number of studies ${ }^{(6,16,17,42,47)}$; however, it does not differentiate between gross and metabolisable energy, which is dependent on the bioavailability of each macronutrient in the food source. This difference can result in an overestimation of energy based on inaccuracies in each macronutrient (protein $5.7 \mathrm{~kJ} / \mathrm{g}(1.36 \mathrm{kcal} / \mathrm{g}$ ), lipids $1.92 \mathrm{~kJ} / \mathrm{g}$ $(0.46 \mathrm{kcal} / \mathrm{g})$ and carbohydrate $0.33 \mathrm{~kJ} / \mathrm{g}(0.08 \mathrm{kcal} / \mathrm{g}))^{(18,30,71)}$, which is important when considering the energy value, the PER of milk feeds and the energy intake of preterm infants. It is common to quantify energy content through the use of factors representing the energy contribution of each macronutrient. Anderson et $a l^{(18)}$, Atkinson et $a l^{(33)}$, Faerk et al. ${ }^{(38)}$, Jitta et $a l .{ }^{(40)}$, Maas et $a l^{(44)}$ and Chessex et al. ${ }^{(36)}$ have used the factors of $23.64,38.70$ and $16.52 \mathrm{~kJ} / \mathrm{g}(5.65,9.25$ and $3.95 \mathrm{kcal} / \mathrm{g})$ for protein, fat and lactose, respectively, which are based on heats of combustion and comparable with bomb calorimetry values $^{(18,71)}$. Saarela et $a l .{ }^{(45)}$ used the Atwater general factor system, which represents the metabolisable energy of protein, fat and carbohydrate with the factors 17,38 and $17 \mathrm{~kJ} / \mathrm{g}$ (4, 9 and $4 \mathrm{kcal} / \mathrm{g})$, respectively ${ }^{(30,72)}$. These factors were further developed to be food specific, forming the Atwater specific factor system $^{(30)}$. This system was used by Gross et al. ${ }^{(20)}$, with the factors for metabolisable energy in milk being $17 \cdot 86,36 \cdot 78$ and $16.19 \mathrm{~kJ} / \mathrm{g}(4.27,8.79$ and $3.87 \mathrm{kcal} / \mathrm{g})$ for protein, fat and 
carbohydrate, respectively ${ }^{(30)}$. Many authors have used lactose concentration in place of carbohydrate when calculating energy content ${ }^{(18,20,33,40,45)}$, because of lactose being the primary carbohydrate in milk, and a better reflection of the carbohydrate component that is digestible, with the remaining component being largely indigestible oligosaccharides. Although the Atwater factor systems are clinically relevant methods of energy determination, there is also the larger measurement error to consider when using factors, as the percentage errors involved in the measurement of individual components of the milk combine to give a much larger error for the derived energy content. As expected, reported energy values based on digestible or metabolisable energy factors were consistently lower than those based on total energy intake values.

\section{Limitations of this review}

Although the authors made every effort to procure papers, a small number of articles that could not be excluded by title or abstract could not be obtained for use in this review. Chemical analyses used in some studies were incompletely described or cited inaccessible reference material; these studies were mentioned within the discussion; however, the validity of the results could not be appreciated. The graphical representation of data without numerical support was a barrier to accurate interpretation. Rather than exclude these studies, the four reviewers independently extracted values manually and reached a consensus value for each relevant weekly average. However, there were many fundamental differences between studies, including number of mothers included, various maternal factors (including age, parity, nutritional and socioeconomic status), gestational ages of the infants and methodologies used, which could not be controlled for and may have contributed to the large range of values identified in the literature.

\section{Conclusion}

This review has provided a compilation of the published values of preterm human milk composition, and has suggested preferred reference values for the assumed macronutrient composition of preterm milk for week 1 (colostrum) and weeks 2-8 of lactation (mature milk). We have calculated estimated macronutrient and energy intakes of infants at prescribed fluid volumes using the minimum, mean and maximum reference values for mature milk and routine fortification practices, as well as demonstrated that recommended protein targets are likely unachievable below volume intakes of $170-190 \mathrm{ml} / \mathrm{kg}$ per $\mathrm{d}$ when the milk has low and average protein content. We have also demonstrated that these fortified preterm milk feeds are unlikely to have an adequate PER, potentially compromising adequate growth. Given the variable composition of breast milk and the fact that preterm infants are a heterogeneous population, different strength fortifiers may be required to meet the needs of very low weight or poorly growing infants. Global acceptance of the recommended preferred reference values for preterm milk composition may ensure standardised calculation and reporting of nutritional intakes, better direct clinicians, researchers and industry in the development of appropriate formulations of $\mathrm{BMF}$, and guide the level of fortification required to better achieve preterm nutrition and growth targets.

None of the studies included in this review used the most accurate methods for measuring each macronutrient in preterm human milk. In order to determine the most accurate preterm breast milk composition, analyses must be undertaken of all macronutrients in 24-h collections from a large sample of healthy mothers over the course of lactation, using the best methodologies available.

\section{Acknowledgements}

The authors acknowledge the support and resources provided by King Edward Memorial Hospital, The University of Western Australia and the ChemCentre at Curtin University.

This research received no specific grant from any funding agency or from commercial or not-for-profit sectors.

C. B., M. W., G. L. and S. R.: equal contribution to the formulation of the research question and study design, collation and review of data, synthesis of results and compilation of the review; K. D.: provision of expert advice on analytical chemistry and assistance in developing the method ranking system; K. S.: contribution to the formulation of the research question and study design and editorial assistance; G. M.: supervisor of the project. Contribution to the development of the research question and study design and provision of expert advice and editorial assistance.

None of the authors has any conflicts of interest to declare.

\section{References}

1. Ehrenkranz R, Younes N, Lemons J, et al. (1999) Longitudinal growth of hospitalized very low birth weight infants. Pediatrics 104, 280-289.

2. Ramel S, Gray H, Ode K, et al. (2011) Body composition changes in preterm infants following hospital discharge: comparison with term infants. J Pediatr Gastroenterol Nutr $\mathbf{5 3}$, 333-338.

3. Parkinson JRC, Parkinson MJ, Hyde C, et al. (2013) Preterm birth and the metabolic syndrome in adult life: a systematic review and meta-analysis. Pediatrics 131, 1240-1263.

4. National Health and Medical Research Council (2013) Infant feeding guidelines: summary. www.nhmrc.gov.au (accessed June 2013).

5. Eidelman A \& Schanler R (2012) Breastfeeding and the use of human milk. Pediatrics 129, e827-e841.

6. Bauer J \& Gerss J (2011) Longitudinal analysis of macronutrients and minerals in human milk produced by mothers of preterm infants. Clin Nutr 30, 215-220.

7. Arslanoglu S, Moro G, Ziegler E, et al. (2010) Optimization of human milk fortification for preterm infants: new concepts and recommendations. J Perinat Med 38, 233-238.

8. Agostoni C, Buonocore G, Carnielli V, et al. (2010) Enteral nutrient supply for preterm infants: commentary from the European Society for Paediatric Gastroenterology, Hepatology, and Nutrition Committee on Nutrition. I Pediatr Gastroenterol Nutr 50, 85-91.

9. Arslanoglu S, Moro G \& Ziegler E (2009) Preterm infants fed fortified human milk receive less protein than they need. J Perinatol 29, 489-492. 
10. Kociszewska-Najman B, Borek-Dzieciol B, SzpotanskSikorska M, et al. (2012) The creamatocrit, fat and energy concentration in human milk produced by mothers of preterm and term infants. J Matern Fetal Neonatal Med 25, 1599-1602.

11. Sauer C \& Kim J (2011) Human milk macronutrient analysis using point-of-care near-infrared spectrophotometry. J Perinatol 31, 339-343.

12. Casadio Y, Williams T, Lai C, et al. (2010) Evaluation of a midinfrared analyzer for the determination of the macronutrient composition of human milk. J Hum Lact 26, 376-383.

13. Corvaglia L, Battistini B, Paoletti V, et al. (2008) Near-infrared reflectance analysis to evaluate the nitrogen and fat content of human milk in neonatal intensive care units. Arch Dis Child Fetal Neonatal Ed 93, F372-F375.

14. Butte N, Garza C, Johnson C, et al. (1984) Longitudinal changes in milk composition of mothers delivering preterm and term infants. Early Hum Dev 9, 153-162.

15. Menjo A, Mizuno K, Murase M, et al. (2009) Bedside analysis of human milk for adjustable nutrition strategy. Acta Paediatr 98, 380-384.

16. Lemons J, Moye L, Hall D, et al. (1982) Differences in the composition of preterm and term human milk during early lactation. Pediatr Res 16, 113-117.

17. Anderson DM, Williams FH, Merkatz RB, et al. (1983) Length of gestation and nutritional composition of human milk. Am J Clin Nutr 37, 810-814.

18. Anderson GH, Atkinson SA \& Bryan MH (1981) Energy and macronutrient content of human milk during early lactation from mothers giving birth prematurely and at term. Am J Clin Nutr 34, 258-265.

19. Weber A, Loui A, Jochum F, et al. (2001) Breast milk from mothers of very low birthweight infants: variability in fat and protein content. Acta Paediatr 90, 772-775.

20. Gross SJ, Geller J \& Tomarelli RM (1981) Composition of breast milk from mothers of preterm infants. Pediatrics $\mathbf{6 8}$, 490-493.

21. Atkinson SA, Anderson GH \& Bryan MH (1980) Human milk: comparison of the nitrogen composition in milk from mothers of premature and full-term infants. Am J Clin Nutr 33, 811-815.

22. Lonnerdal B (2003) Nutritional and physiologic significance of human milk proteins. Am J Clin Nutr 77, 1537S-1543S.

23. Jensen RG (1999) Lipids in human milk. Lipids 34, 1243-1271.

24. Khan S, Prime DK, Hepworth AR, et al. (2013) Investigation of short-term variations in term breast milk composition during repeated breast expression sessions. J Hum Lact 29, 196-204.

25. Fusch G, Rochow N, Choi A, et al. (2015) Rapid measurement of macronutrients in breast milk: how reliable are infrared milk analyzers? Clin Nutr 34, 465-476.

26. Smilowitz JT, Gho DS, Mirmiran M, et al. (2014) Rapid measurement of human milk macronutrients in the neonatal intensive care unit: accuracy and precision of Fourier transform mid-infrared spectroscopy. J Hum Lact 30, 180-189.

27. Gidrewicz DA \& Fenton TR (2014) A systematic review and meta-analysis of the nutrient content of preterm and term breast milk. BMC Pediatr 14, 216-229.

28. International Organization for Standardization (2005) ISO/IEC 17025:2005. General requirements for the competence of testing and calibration laboratories. http://www.iso.org/ (accessed August 2015).

29. Bradstreet R (1965) The Kjeldahl Method for Organic Nitrogen. New York: Academic Press.

30. Food and Agriculture Organization of the United Nations (2003) Food energy - methods of analysis and conversion factors. www.fao.org (accessed September 2013).
31. Fomon SJ (1991) Requirements and recommended dietary intakes of protein during infancy. Pediatr Res 30, 391-395.

32. Atkinson SA, Bryan MH \& Anderson GH (1978) Human milk: difference in nitrogen concentration in milk from mothers of term and premature infants. J Pediatr 93, 67-69.

33. Atkinson SA, Bryan MH \& Anderson GH (1981) Human milk feeding in premature infants: protein, fat, and carbohydrate balances in the first two weeks of life. J Pediatr 99, 617-624.

34. Beijers RJ, Graaf FV, Schaafsma A, et al. (1992) Composition of premature breast-milk during lactation: constant digestible protein content (as in full term milk). Early Hum Dev 29, 351-356.

35. Beijers RJ \& Schaafsma A (1996) Long-chain polyunsaturated fatty acid content in Dutch preterm breast milk; differences in the concentrations of docosahexaenoic acid and arachidonic acid due to length of gestation. Early Hum Dev 44, 215-223.

36. Chessex P, Reichman B, Verellen G, et al. (1983) Quality of growth in premature infants fed their own mothers' milk. J Pediatr 102, 107-112.

37. Ehrenkranz RA, Ackerman BA \& Nelli CM (1984) Total lipid content and fatty acid composition of preterm human milk. J Pediatr Gastroenterol Nutr 3, 755-758.

38. Faerk J, Skafte L, Petersen S, et al. (2001) Macronutrients in milk from mothers delivering preterm. Adv Exp Med Biol 501, 409-413.

39. Guerrini P, Bosi G, Chierici R, et al. (1981) Human milk: relationship of fat content with gestational age. Early Hum Dev 5, 187-194.

40. Jitta JN, Musoke RN, Bwibo NO, et al. (1986) Composition of early human milk of Kenyan mothers of preterm and term infants. East Afr Med J 63, 693-698.

41. Lemons JA, Reyman D \& Moye L (1983) Amino acid composition of preterm and term breast milk during early lactation. Early Hum Dev 8, 323-329.

42. Lepage G, Collet S, Bougle D, et al. (1984) The composition of preterm milk in relation to the degree of prematurity. Am J Clin Nutr 40, 1042-1049.

43. Lucas A \& Hudson GJ (1984) Preterm milk as a source of protein for low birthweight infants. Arch Dis Child 59, 831-836.

44. Maas YG, Gerritsen J, Hart AA, et al. (1998) Development of macronutrient composition of very preterm human milk. Br J Nutr 80, 35-40.

45. Saarela T, Kokkonen J \& Koivisto M (2005) Macronutrient and energy contents of human milk fractions during the first six months of lactation. Acta Paediatr 94, 1176-1181.

46. Sann L, Bienvenu F, Lahet C, et al. (1981) Comparison of the composition of breast milk from mothers of term and preterm infants. Acta Paediatr Scand 70, 115-116.

47. Silber GH, Hachey DL, Schanler RJ, et al. (1988) Manipulation of maternal diet to alter fatty acid composition of human milk intended for premature infants. Am J Clin Nutr 47, 810-814.

48. Stevens LH (1969) The first kilogram. 2. The protein content of breast milk on mothers of babies of low birth weight. Med J Aust 2, 555-557.

49. National Health and Medical Research Council (2006) Nutrient reference values for Australia and New Zealand. www.nrv. gov.au (accessed April 2016).

50. Horbar JD, Ehrenkranz RA, Badger GJ, et al. (2015) Weight growth velocity and postnatal growth failure in infants 501 to 1500 grams. Pediatrics 136, e84-e92.

51. Tsang RC, Uauy R, Koletzko B, et al. (2005) Nutrition of the Preterm Infant. Scientific Basis and Practical Guidelines, 2nd ed. Cincinnati, OH: Digital Educational Publishing Inc. 
52. Polberger S, Raiha NC, Juvonen $\mathrm{P}$, et al. (1999) Individualised protein fortification of human milk for preterm infants: comparison of ultrafiltrated human milk protein and bovine whey fortifier. J Pediatr Gastroenterol Nutr 29, 332-338.

53. McLeod G, Sherriff J, Hartmann PE, et al. (2016) Comparing different methods of human breast milk fortification using measured $\mathrm{v}$. assumed macronutrient composition to target reference growth: a randomised controlled trial. BrJ Nutr $\mathbf{1 1 5}$, $431-439$.

54. Miller J, Makrides M, Gibson RA, et al. (2012) Effect of increasing protein content of human milk fortifier on growth in preterm infants born at $<31 \mathrm{wk}$ gestation: a randomised controlled trial. Am J Clin Nutr 95, 648-655.

55. Sherriff J \& McLeod G (2013) Breast milk additives and infant formula: a clinical perspective. In Nutrition for the Preterm Neonate, pp. 156-158 [S Patole, editor]. Dordrecht: Springer.

56. Ziegler EE (2011) Meeting the nutritional needs of the low-birth-weight infant. Ann Nutr Metab 58, 8-18.

57. Donovan S \& Lonnerdal B (1989) Development of a human milk protein standard. Acta Paediatr Scand 78, 171-179.

58. Bergqvist Y, Karisson L \& Fohlin L (1989) Total protein determined in human breast milk by use of Coomassie Brilliant Blue and centrifugal analysis. Clin Chem 35, 2127-2129.

59. Lonnerdal B, Woodhouse L \& Glazier C (1987) Compartmentalization and quantitation of protein in human milk. J Nutr 117, 1385-1395.

60. Keller R \& Neville M (1986) Determination of total protein in human milk: comparison of methods. Clin Chem 32, 120-123.

61. Okutucu B, Dinçer A, Habib O, et al. (2007) Comparison of five methods for determination of total plasma protein concentration. J Biochem Biophys Methods 70, 709-711.
62. Lewis B \& Richards P (1961) Measurement of urinary protein. Lancet 277, 1141-1143.

63. Agelet L \& Hurburgh C Jr (2010) A tutorial on near infrared spectroscopy and its calibration. Crit Rev Anal Chem 40, 246-260.

64. Jensen R \& Clark R (1984) Methods of lipid analysis. J Pediatr Gastroenterol Nutr 3, 296-299.

65. Horwitz W (1975) Official Methods of Analysis of the Association of Official Analytical Chemists, 13th ed. Washington, DC: Association of Official Analytical Chemists.

66. Insull W Jr \& Ahrens EH Jr (1959) The fatty acids of human milk from mothers on diets taken ad libitum. Biochem $J \mathbf{7 2}$, 27-33.

67. Frings C \& Dunn R (1970) A colorimetric method for determination of total serum lipids based on the sulfophospho-vanillin reaction. Am J Clin Pathol 53, 89-91.

68. Lucas A, Gibbs J, Lyster R, et al. (1978) Creamatocrit: simple clinical technique for estimating fat concentration and energy value of human milk. BMJ 1, 1018-1020.

69. García-Lara N, Escuder-Vieco D, García-Algar O, et al. (2012) Effect of freezing time on macronutrients and energy content of breastmilk. Breastfeed Med 7, 295-301.

70. Coppa G, Gabrielli O, Pierani P, et al. (1993) Changes in carbohydrate composition in human milk over 4 months of lactation. Pediatrics 91, 637-641.

71. Neville M (1995) Determinants of milk volume and composition: B. Volume and caloric density of human milk. In Handbook of Milk Composition, pp. 108-109 [R Jensen, editor]. Burlington: Elsevier.

72. Southgate D \& Barrett I (1966) The intake and excretion of calorific constituents of milk by babies. Br J Nutr 20, 363-372. 